\title{
ESTIMACIÓN DEL CONTENIDO DE AGUA A PARTIR DE MEDICIONES HIPERESPECTRALES PARA CARTOGRAFÍA DEL RIESGO DE INCENDIO
}

\author{
M.E. HERRERA, E. CHUVIECO* \\ Grupo de Teledetección Ambiental, Departamento de Geología, Geografía y Medio Ambiente, \\ Universidad de Alcalá, Colegios 2, Alcalá de Henares, 28801 Madrid.
}

\begin{abstract}
RESUMEN. Este trabajo pretende mostrar el interés de las mediciones hiperespectrales de laboratorio para estimar el contenido de humedad de cara a obtener esta variable operativamente en el contexto de la determinación del riesgo de incendio. El experimento se ha realizado con dos especies forestales mediterráneas (alcornoque y roble), que fueron sometidas a un proceso de estrés hídrico controlado. Las mediciones hiperespectrales se realizaron con una cámara Specim, sensible a las longitudes de onda entre 900 y $1700 \mathrm{~nm}$. A partir de estas mediciones, se calculó la correlación espectral con las variaciones del contenido de humedad, tanto para las bandas de la cámara empleada, como para las correspondientes al sensor MODIS (Moderate Resolution Imaging Spectroradiometer). Para el índice espectral Normalized Difference Water Index (NDWI) se obtuvieron valores altos de correlación, tanto para el $\mathrm{Q}$. suber $(r=0.803$ con el Fuel Moisture Content, FMC y $r=0.846$ con el Equivalent Water Thickness, EWT), como para el Q. robur ( $r=0.705$ con el FMC y $r=0.802$ para EWT). Esto confirma que ambos parámetros pueden estimarse con adecuada precisión usando imágenes de satélite.
\end{abstract}

Estimating water content from hyperspectral measurements for fire risk mapping

ABSTRACT. This paper shows the interest of laboratory hyperspectral measurements to estimate fuel moisture content for fire risk assessment. Measurements were obtained for two Mediterranean forest species over a controlled water stress experiment. The hyperspectral measurements were performed with a Specim camera, sensible to spectral reflectance from 900 to $1700 \mathrm{~nm}$. We calculated the spectral correlation with variations in the moisture content, both for the camera raw bands as well as for those adjusted to the MODIS (Moderate Resolution Imaging Spectroradiometer) sensor. High correlation values were obtained for moisture content measurements and the Normalized Difference Water Index (NDWI), both for Q. suber $(r=0.803$ with the FMC and $r=0.846$ for EWT), and for the Q. robur $(r=0.705$ with the FMC and 
$r=0.802$ for EWT). Our work asserts that both parameters can be estimated with appropriate accuracy using satellite images.

Palabras clave: contenido de humedad del combustible, espesor específico de agua, MODIS.

Key words: fuel moisture content, equivalent water thickness, MODIS.

Enviado el 13 de noviembre de 2013

Aceptado el 13 de febrero de 2014

Correspondencia: Grupo de Teledetección Ambiental, Departamento de Geología, Geografía y Medio Ambiente, Universidad de Alcalá, Colegios 2, Alcalá de Henares, 28801 Madrid. E-mail: emilio.chuvieco@uah.es

\section{Introducción}

El contenido de humedad de la vegetación es un aspecto de gran importancia en el funcionamiento fisiológico de las plantas, afectando a la productividad de los ecosistemas, la evapotranspiración, el crecimiento celular y la fotosíntesis (Boyer, 1995). En el ámbito de los incendios forestales, la medida más habitual de contenido de humedad es el denominado FMC (Fuel Moisture Content), definido como la cantidad de agua en función de la masa de la muestra:

$$
F M C_{(\%)}=\frac{W f-W s}{W s} \times 100
$$

donde $W f$ es el peso fresco y $W s$ el peso seco.

El FMC es un parámetro de gran relevancia para estimar la probabilidad de ignición o propagación del fuego, puesto que afecta al calor necesario para que una determinada especie arda (Burgan y Rothermel, 1984). En la literatura especializada, el FMC se ha calculado a partir de diversos métodos. La estimación directa se realiza mediante el método gravimétrico, por diferencia de pesos tras secar las muestras en estufa a una temperatura constante (habitualmente $24 \mathrm{~h} \mathrm{a} 60^{\circ} \mathrm{C}$ ). Las mediciones se realizan a partir de muestreos en campo, normalmente repetidos con una cierta cadencia temporal (de diaria a quincenal es lo más común). Este enfoque es preciso, pero muy costoso y habitualmente poco representativo espacialmente (Chuvieco et al., 2004). En la práctica es utilizado para calibrar o validar otras metodologías (Yebra et al., 2013).

Como parte de los métodos indirectos se utilizan los índices meteorológicos de peligro (Castro et al., 2003) que funcionan bien con combustibles muertos, pero no tanto en vivos, ya que se asume una respuesta uniforme de las distintas especies a la variación de las condiciones atmosféricas (Camia et al., 2003), sin considerar los diferentes mecanismos que las plantas tienen para resistir a la carencia de agua. Adicionalmente, las estaciones meteorológicas no siempre están próximas a áreas forestales, ni tienen la densidad adecuada, y el contenido de agua de las plantas no sólo depende de las condiciones 
atmosféricas sino también de las características fisiológicas de la vegetación (Ceccato et al., 2003).

La estimación del FMC para los combustibles vivos (a partir de ahora nos referimos exclusivamente a la humedad de éstos) también se puede realizar mediante el uso de imágenes satelitales, basándose en la relación existente entre la información espectral y el estado hídrico de la vegetación (Yebra et al., 2013). El uso de las imágenes satelitales es una de las formas más prácticas de monitorear la vegetación a escalas locales y globales, proporcionando una cobertura sistemática y espacialmente completa.

La estimación del FMC a partir de imágenes se ha abordado mediante ajustes empíricos o modelos de simulación (Yebra et al., 2013). Los primeros son sencillos de calibrar, pero requieren mediciones del FMC en terreno, simultáneas al paso del satélite (Yebra et al., 2008). Los modelos de simulación son más complejos de calibrar, pero tienen mayor poder de generalización (Yebra et al., 2013). Habitualmente se basan en la ecuación de transferencia radiativa (Radiative Transfer Models, RTM), que considera los distintos elementos absorbentes y dispersores en la planta. Los RTM pueden plantearse en modo directo, variando los parámetros de entrada para observar cómo cambia la reflectividad simulada, o inverso, a partir de la reflectividad medida por el sensor para estimar qué condiciones de humedad dieron lugar a esa reflectividad simulada. Para obtener estimaciones precisas, estos modelos deben ser adecuadamente parametrizados, para evitar efectos de inversión no singular (Yebra y Chuvieco, 2009).

Para estimar el contenido de agua mediante teledetección, en ocasiones no se utiliza la medida del FMC, sino el denominado EWT (Equivalent Water Thickness), que indica el espesor óptico del agua y se obtiene a partir de la cantidad de agua y la superficie foliar:

$$
E W T\left(g / \mathrm{cm}^{2}\right)=\frac{W f-W s}{A}
$$

Donde $W f$ es el peso fresco, $W s$ el peso seco y A, el área de la hoja. La relación entre FMC y EWT viene dada por el contenido de materia seca de la muestra (DMC, Dry Matter Content):

$$
\operatorname{DMC}\left(\mathrm{g} / \mathrm{cm}^{2}\right)=\frac{W S}{A}
$$

En resumen el FMC puede también expresarse como:

$$
F M C_{(\%)}=\frac{E W T}{D M C} \times 100
$$

Para estimar cualquiera de estas dos medidas de humedad, FCM o EWT, varios autores han sugerido el interés de realizar medidas espectrales en condiciones controladas, mediante espectro-radiómetros de laboratorio. Lo más habitual es que se trate de equipos unidimensionales, que miden la reflectividad en un punto singular, para una 
gran cantidad de longitudes de onda (Bowyer y Danson, 2004). En el marco de nuestro grupo de investigación, se realizó un experimento comparando las medidas de uno de estos radiómetros con una cámara hiperespectral, de cara a estimar el contenido de agua en hojas de Quercus ilex. Se analizó también cómo variaban sus parámetros biofísicos y bioquímicos ante el estrés hídrico al que se sometieron las plántulas en condiciones controladas (De Santis et al., 2006). En este trabajo nos proponemos ampliar ese experimento, incluyendo dos especies de roble muy común en los ecosistemas mediterráneos (Q. robur y $Q$. suber), además de ensayar la aplicación de distintos índices espectrales del sensor MODIS a la determinación del FMC y del EWT. El trabajo parte de un experimento de laboratorio, que se realizó a partir de la misma cámara hiperespectral empleada por De Santis et al. (2006), garantizando una continuidad temporal en las medidas realizadas bajo diferentes condiciones de estrés hídrico.

\section{Metodología}

\subsection{Especies seleccionadas}

Las especies seleccionadas para el desarrollo del experimento fueron el alcornoque (Q. suber) y el roble albar ( $Q$. robur). Ambas son bastante comunes en España y tienen mucho interés desde el punto de vista de su respuesta al fuego. Completan otras medidas que nuestro grupo de investigación ha realizado sobre especies coníferas y de matorral (Jurdao et al., enviado).

El alcornoque ( $Q$. suber) es una especie endémica de la región mediterránea occidental, aparece silvestre en Portugal, España, sur de Francia, Marruecos, Argelia, Túnez, Córcega y Cerdeña, Italia, Eslovenia, Croacia y Bosnia-Herzegovina. Se conservan actualmente en el mundo algo más de dos millones de hectáreas de alcornocales, aportando el máximo Portugal, seguido de Argelia y España. En España aparece en el cuadrante suroccidental: Sierra Morena, Extremadura y Montes de Toledo, sobre una superficie de unas 311000 ha, de las cuales 231000 están sobre suelo forestal y 80000 sobre suelo de dedicación mixta con cultivo agrícola (Ruiz de la Torre, 1990-1999). El alcornoque es poco xerófilo, requiriendo cierta humedad en el ambiente y más bien termófilo. Prefiere terreno suelto y fresco y algo húmedo en profundidad, pero no encharcado. Necesita una precipitación media anual superior a $400 \mathrm{~mm}$.

El roble albar ( $Q$. robur) es una especie forestal dominante en Europa central y occidental, que alcanza su límite suroccidental de distribución en el noroeste de la Península Ibérica. En este territorio se han identificado cinco grupos geográficamente diferenciados de patrones de crecimiento y respuesta climática (Méndez y Molinero, 1993): Sierras Nororientales, con efecto positivo de la temperatura en mayo y septiembre; tierras bajas y Sierras Centrales, con efecto negativo de la temperatura y positivo de la precipitación en junio; costa norte con ausencia de condiciones climáticas restrictivas para el crecimiento; costa Sur, con efecto negativo de la temperatura en diciembre y positivo de la precipitación en agosto, e interior Sur, con efecto positivo de la precipitación en invierno (noviembre, diciembre) y primavera-verano (desde mayo hasta agosto). Los tres primeros grupos presentan una respuesta climática esperable bajo clima atlántico litoral 
o de montaña, sin limitaciones severas al crecimiento por causas climáticas. Los dos últimos crecen bajo clima con tendencias mediterráneas, reflejando una mayor sensibilidad del crecimiento a la sequía estival. En caso de una eventual acentuación de la intensidad y duración del período de sequía estival las poblaciones del sur mostrarían una mayor reducción de la vitalidad y crecimiento.

\subsection{Parámetros biofísicos medidos}

Las mediciones de laboratorio se realizaron con veinte plántulas de dos años de edad, diez de ellas de $Q$. robur y diez de $Q$. suber, de entre ellas se seleccionaron cuatro plántulas para cada especie, de tal forma que presentaran las características más homogéneas y con valor más alto de área foliar. El resto de plántulas fueron utilizadas como control. Una vez seleccionadas las plántulas, fueron irrigadas una única vez al inicio del experimento (6 de mayo de 2013), y dejadas posteriormente sin agua hasta que la pérdida de humedad fuera muy evidente, midiendo los parámetros indicados diariamente sobre las hojas extraídas de las plántulas en cada medición. El proceso de mediciones se inició el 14 de mayo y finalizó el 14 de junio, utilizando el método gravimétrico para estimación del FMC. Un fallo técnico en la cámara hiperespectral impidió realizar mediciones entre los días 15 y 29 de mayo. Tampoco pudieron hacerse los fines de semana. Se procuró que en el proceso las plántulas no estuvieran sometidas a estrés lumínico y tuvieran la suficiente aireación de manera que sus condiciones fisiológicas no se viesen afectadas por factores diferentes al hídrico. Los parámetros medidos fueron la reflectividad en diversas bandas del espectro (950 a $1677 \mathrm{~nm}$ ), el FMC, EWT y DMC.

El equipo utilizado para las mediciones radiométricas fue la cámara hiperespectral Specim, que incluye un espectrógrafo manufacturado por Specim, Oulu, Finlandia (http://www. specim.fi), que permite descomponer la energía reflejada desde la muestra en 166 bandas espectrales, entre 950 a 1677 nm (rango oficial 900 - $1700 \mathrm{~nm}$ ). El área observada es de 320 píxeles (FOV de $24^{\circ}$ en total $-0.075^{\circ}$ por píxel). La imagen se forma por un espejo oscilante, que barre una superficie perpendicular a la posición de la cámara.

Para las mediciones de los parámetros biofísicos se utilizó una balanza de precisión GRAM serie VSI-650 con una precisión de $\pm 0.001 \mathrm{~g}$, el software Winfolia (Regent Instruments Inc, Canada), un escáner Epson para la medición de la superficie de las hojas y una estufa para el secado.

En cada día de medición se recolectaron dos hojas por cada plántula de ambas especies. Una vez realizada la medición de reflectividad de las hojas, éstas fueron pesadas en la balanza para obtener el peso fresco de cada una, la medición de la superficie se realizó escaneando dichas hojas para su posterior procesamiento con el software Winfolia. Una vez medidos ambos parámetros, las muestras se secaron en una estufa durante 24 horas a temperatura de $100 \pm 5^{\circ} \mathrm{C}$ hasta tener un peso constante, lo que indica que están completamente secas. En ese momento fueron pesadas de nuevo para obtener su peso seco (Fig. 1). Con los valores de peso fresco, peso seco y superficie de cada muestra de hoja se calcularon los parámetros biofísicos, tal y como se indica en las fórmulas anteriormente incluidas $(1,2,3)$. 


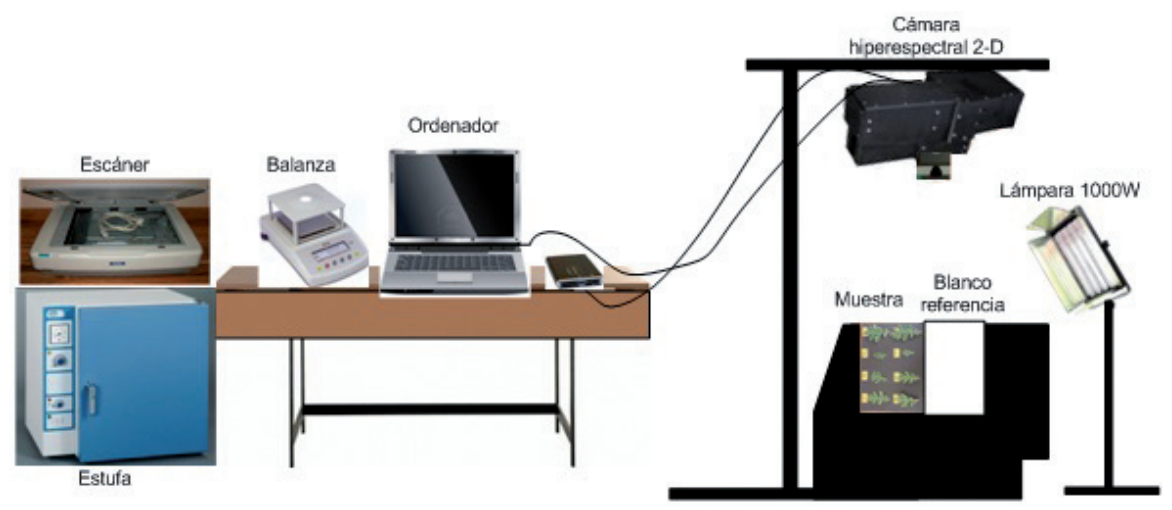

Figura 1. Esquema del procedimiento completo para las mediciones de laboratorio.

\subsection{Mediciones de Reflectividad}

La reflectividad de las hojas fue medida utilizando la cámara hiperespectral Specim antes descrita. Se instaló la cámara sobre su respectivo soporte para mantener constantes las características de posición y orientación en cada medición, se acondicionó el espacio de las mediciones para minimizar el efecto de la radiación difusa en la reflectividad medida, rodeando las muestras de una estructura móvil pintada de negro. Empleamos como fuente de luz una lámpara de $1000 \mathrm{~W}$, colocada sobre un trípode para mantener su orientación y posición constantes a lo largo de todas las mediciones. Fue encendida con un tiempo mínimo de 30 minutos antes de cada medición.

Para cada medición se colocaron las muestras en paralelo al blanco de referencia (Fig. 1), de manera tal que las mediciones se pudieran convertir en reflectividad al dividir el valor de radiancia de las muestras, por las obtenidas en el blanco de referencia (De Santis et al., 2006). De cada hoja se tomaron 4 muestras de reflectividad a fin de evitar errores aleatorios o groseros en las mediciones de las mismas, y la reflectividad se calculó como un promedio de las cuatro mediciones.

\section{4. Índices espectrales para la estimación del FMC}

Junto a la relación entre las mediciones hiperespectrales y los parámetros de las hojas, calculamos algunos índices espectrales que se han propuesto en la literatura para la estimación del contenido de agua. En concreto empleamos el Normalized Difference Water Index - NDWI (Gao, 1996), que tiene en cuenta la absorción diferencial del agua en el infrarrojo cercano y de onda corta:

$$
N D W I=\frac{(\rho 860-\rho 1240)}{(\rho 860+\rho 1240)}
$$

Este índice se calculó a partir de las reflectividades medidas por el sistema Specim. Como no teníamos disponibles la banda $860 \mathrm{~nm}$ en nuestra cámara, decidimos adaptar el 
índice a algunas bandas que incluye el sensor MODIS, uno de los más interesantes para la aplicación operativa de la teledetección en la estimación del agua. Se utilizaron las bandas $5(1230-1250 \mathrm{~nm}), 6(1628-1652 \mathrm{~nm})$ y 19 (915-965 nm), calculándose los siguientes índices:

$$
\begin{gathered}
N D W I_{t 19,6}=\frac{(\rho 937-\rho 1640)}{(\rho 937+\rho 1640)} \\
N D W I_{t 5,6}=\frac{(\rho 1240-\rho 1640)}{(\rho 1240+\rho 1640)}
\end{gathered}
$$

\subsection{Análisis estadístico}

Empleamos el coeficiente de Pearson para determinar la relación entre los parámetros biofísicos medidos con la respuesta espectral de las plántulas en los diversos periodos de medida:

$$
r_{x y}=\frac{\sum x_{i} y_{i}}{n S_{x} S_{y}}
$$

Donde $x_{i}$ e $y_{i}$ se refieren a las puntuaciones diferenciales de cada par; $n$ al número de casos y $S_{x}$ y $S_{y}$ a las desviaciones típicas de cada variable.

También elaboramos un modelo de ajuste multivariable, que permitiera estimar el FMC o EWT a partir de las reflectividades medidas en la cámara Specim. El modelo de regresión, sería:

$$
Y=\beta_{0}+\beta_{1} X_{1}+\beta_{2} X_{2}+\ldots \ldots+\beta_{k} X_{k}+\varepsilon
$$

Donde $Y$ sería el EWT o FMC, $X_{k}$ las variables explicativas (en nuestro caso las reflectividades de las bandas significativas) y $\left(\beta_{k}\right)$ son los coeficientes de regresión. La entrada de las variables independientes se realizó a partir de pasos sucesivos con inclusión, señalando un nivel de significancia de 0.05 para la entrada y 0.1 para la salida.

La bondad del ajuste se estimó a partir del $\left(r^{2}\right)$ para determinar la proporción de varianza de la variable dependiente (FMC y EWT) que está explicada por las variables independientes (reflectividades). Este valor se ajustó ( $r^{2}$ corregida) a partir del número de casos y de variables independientes:

$$
r_{\text {corregida }}^{2}=r^{2}-\frac{p\left(1-r^{2}\right)}{(n-p-1)}
$$

Donde $p$ se refiere al número de variables independientes.

El error típico de la estimación $S_{e}$ representa la medida de la variabilidad de la variable dependiente que no esté explicada por la recta de regresión.

$$
S_{e}=\sqrt{\sum \frac{(Y i-\bar{Y} i)^{2}}{(n-2)}}
$$




\section{Resultados}

\subsection{Evolución de los parámetros foliares medidos}

En las Figs. 2 y 3 se observan los resultados promedios de la respuesta de los parámetros biofísicos FMC, EWT y DMC al estrés hídrico, durante los 9 días de mediciones, las curvas muestran claramente una tendencia similar para ambas especies en las que a medida que el estrés hídrico aumenta en la planta, el FMC y EWT disminuyen sus valores, mostrando así una relación inversa. Sin embargo se observa que el DMC casi se mantiene constante a lo largo del experimento mostrando poca variación en su contenido de materia seca, lo que implica que es apenas afectado por los cambios en el contenido de agua.

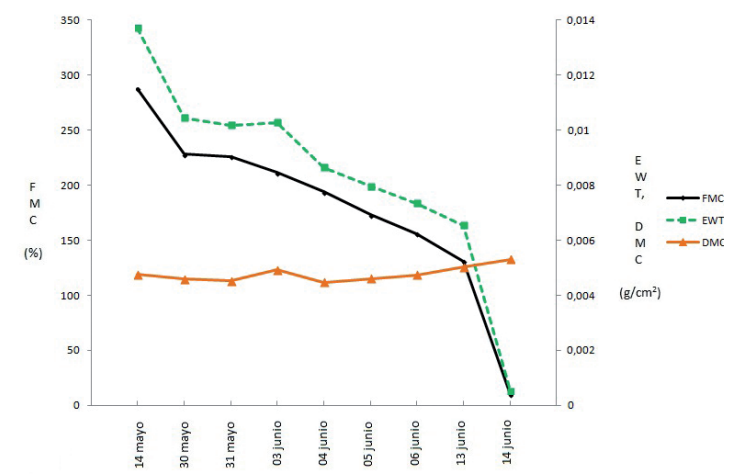

Figura 2. Valores promedio FMC, EWT y DMC (Q. suber).

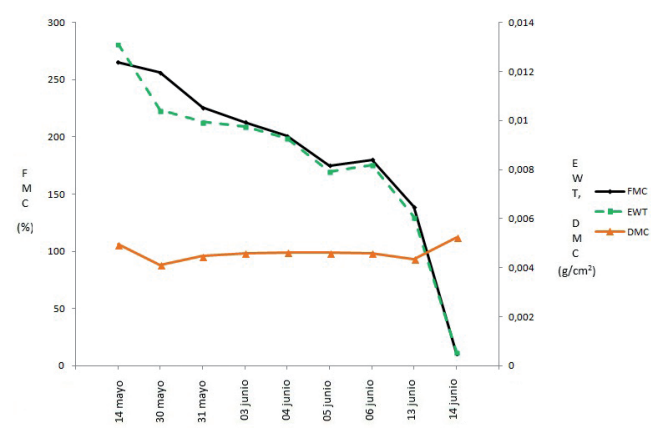

Figura 3. Valores promedio FMC, EWT y DMC (Q. robur).

Se observa que a pesar de que ambas especies tengan la misma tendencia en la respuesta de sus parámetros, los valores de cada parámetro varían ligeramente, debido a que cada planta tiene su propio ritmo de desecación, con valores algo superiores para el $Q$. robur. 


\subsection{Análisis de las variaciones de reflectividad}

Las tendencias de reflectividad de las hojas con el cambio en el contenido de humedad pueden observarse en las Figs. 4 y 5 para varias de las mediciones diarias. Hemos indicado el valor medio de ese día para el FMC. Como era esperable, se observa una variación más nítida de la reflectividad en la banda próxima a los $1450 \mathrm{~nm}$, que es la más adecuada para observar la absorción del agua. En el infrarrojo cercano, ambas especies muestran un aumento, aunque la relación con el contenido de agua es menos nítida, sobre todo en el alcornoque, en donde las últimas mediciones se solapan.

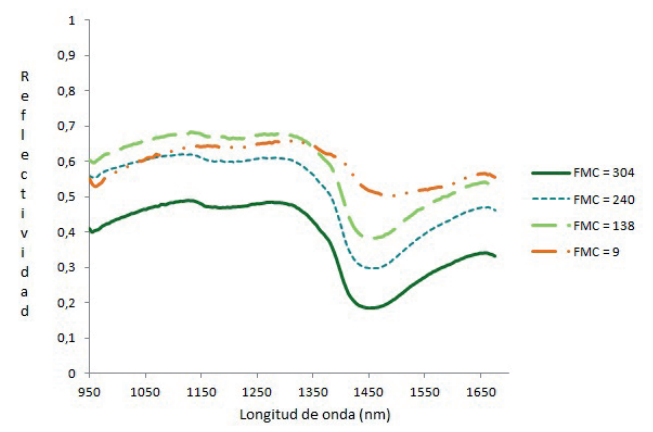

Figura 4. Respuesta espectral a diferentes niveles de FMC, medidos con cámara Specim (Q. suber).

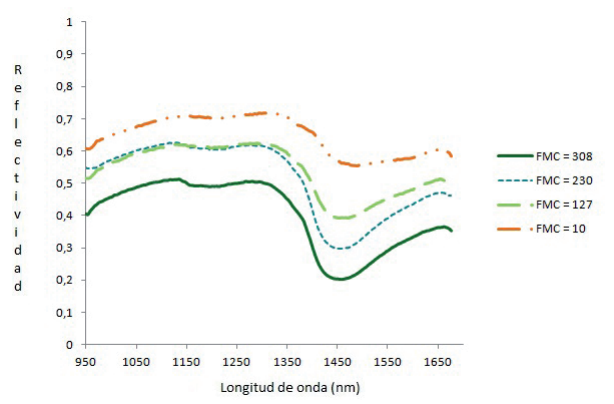

Figura 5. Respuesta espectral a diferentes niveles de FMC, medidos con cámara Specim (Q. robur).

\subsection{Tendencias en la correlación con las reflectividades}

En la tabla 1 se muestran los coeficientes de Pearson entre los parámetros biofísicos medidos en el experimento. Se evidencia una alta correlación positiva entre el FMC y el EWT (0.96 y 0.90) para cada especie, lo que permitiría estimar directamente uno de los parámetros en función del otro. Esto es consecuencia de la escasa variación temporal del DMC, que no se altera significativamente con el secado de la hoja, al menos cuando se hace de manera forzada como es el caso de nuestro experimento. Por esta razón también, la variación temporal del FMC presenta poca relación con la del DMC, siendo muy poco significativa para el EWT. 
Tabla 1. Coeficientes de correlación de Pearson entre los parámetros biofísicos de las hojas.

\begin{tabular}{|l|c|c|c|c|}
\hline & $\begin{array}{c}\text { Q. suber } \\
\mathbf{r}\end{array}$ & $\begin{array}{c}\text { Q. suber } \\
\mathbf{p}\end{array}$ & $\begin{array}{c}\text { Q. robur } \\
\mathbf{r}\end{array}$ & $\begin{array}{c}\text { Q. robur } \\
\mathbf{p}\end{array}$ \\
\hline FMC-EWT & 0.964 & 0.000 & 0.906 & 0.000 \\
\hline FMC-DMC & -0.458 & 0.000 & -0.288 & 0.026 \\
\hline EWT-DMC & -0.228 & 0.089 & 0.123 & 0.348 \\
\hline
\end{tabular}

Los coeficientes de correlación de los parámetros biofísicos con la reflectividad de cada especie aparecen en las Figs. 6 y 7. Se observa claramente que las variaciones del FMC y EWT, tanto para el $Q$. suber como para el $Q$. robur, apenas afectan a la reflectividad de las bandas del infrarrojo cercano hasta $1150 \mathrm{~nm}$, pero son bastante significativas en longitudes mayores. En el caso del $Q$. suber, la correlación se hace más intensa a partir de $1350 \mathrm{~nm}$, siendo máxima hacia $1450 \mathrm{~nm}$, siempre con carácter negativo, ya que el agua supone una absorción de la luz en estas longitudes de onda. Similares tendencias aparecen para el EWT y el FMC. Para el DMC la correlación es mucho más baja, tanto en las bandas del infrarrojo cercano como en las del SWIR, sube un poco aunque en valores muy bajos (máximo 0.2) y tiene, en este caso, un signo positivo.

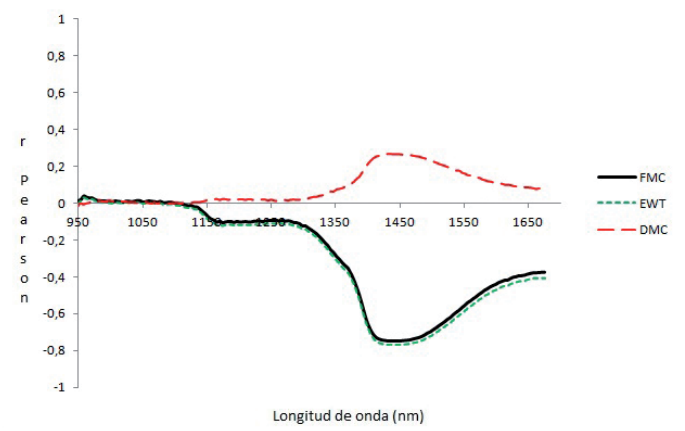

Figura 6. Coeficiente de correlación de Pearson de la reflectividad espectral con el FMC, EWT y $D M C$ (Q. suber).

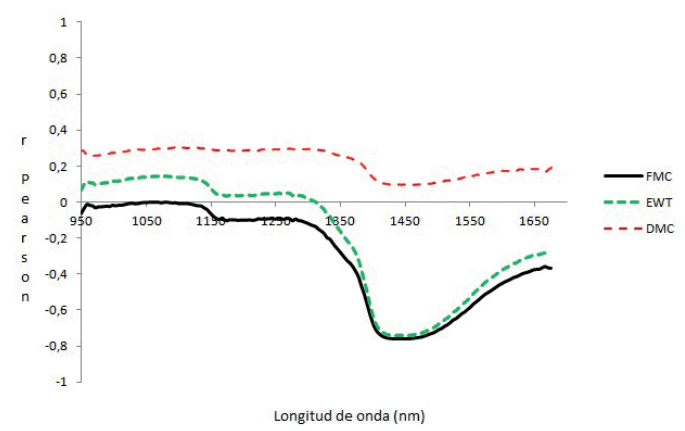

Figura 7. Coeficiente de correlación de Pearson de la reflectividad espectral con el FMC, EWT y $D M C$ (Q. robur). 
Para el caso del $Q$. robur, las tendencias son similares, aunque las correlaciones son un poco más altas y positivas en el infrarrojo cercano, particularmente con el DMC y EWT, aunque siempre por debajo de 0.3. En el SWIR, las hojas de roble, al igual que las del alcornoque, muestran una correlación negativa alta entre FMC y EWT con la reflectividad, particularmente en las bandas de absorción del agua, próximas a $1450 \mathrm{~nm}$. En esta especie, no existe apenas correlación positiva para el DMC en estas regiones.

Estas diferentes respuestas espectrales en las especies pueden ser debidas a sus diferentes formas de reaccionar antes el estrés hídrico y sus consecuencias en la estructura celular interna de la hoja.

\section{4. Índices espectrales}

La relación de los parámetros biofísicos con los índices de estimación de contenido de agua modificado, cuyo fundamento es el uso de la relación entre el IRC y el SWIR, refleja una alta correlación con el FMC y EWT para ambas especies, con valores altamente significativos ( $\mathrm{p}<0.01$ : Tabla 2). Para el DMC, las correlaciones son mucho menores y no significativas, lo cual es esperable, pues estamos empleando bandas que se asocian al agua, pero no a la producción de biomasa. Comparando ambas especies, $Q$. suber presenta mayores correlaciones con los índices modificados, pudiendo concluirse que sería más precisa la detección del contenido de agua para esta especie en relación al roble. Se observa también que el uso del rango espectral de la banda 5 de MODIS tiene mayor correlación con el FMC y el EWT, indicando que es más sensible al contenido de agua que la banda 19. No existen correlaciones buenas con respecto al DMC, por lo que no se podría utilizar este índice para su estimación.

Tabla 2. Coeficiente de correlación de Pearson para los parámetros biofísicos y el NDWIt.

\begin{tabular}{|c|c|c|c|c|}
\hline & $\begin{array}{c}Q . \text { suber } \\
\mathrm{r}\end{array}$ & $\begin{array}{c}Q . \text { suber } \\
\text { p }\end{array}$ & $\begin{array}{c}Q . \text { robur } \\
\mathbf{r}\end{array}$ & $\begin{array}{c}Q . \text { robur } \\
\mathbf{p}\end{array}$ \\
\hline FMC - NDWI ${ }_{t 19.6}$ & 0.803 & 0.000 & 0.705 & 0.000 \\
\hline EWT - NDWI ${ }_{t 19.6}$ & 0.846 & 0.000 & 0.802 & 0.000 \\
\hline DMC - NDWI ${ }_{t 19.6}$ & -0.148 & 0.271 & 0.163 & 0.214 \\
\hline FMC - NDWI ${ }_{t 5.6}$ & 0.820 & 0.000 & 0.735 & 0.000 \\
\hline EWT - NDWI ${ }_{t 5.6}$ & 0.864 & 0.000 & 0.858 & 0.000 \\
\hline DMC - NDWI ${ }_{t 5.6}$ & -0.151 & 0.262 & 0.215 & 0.099 \\
\hline
\end{tabular}

\subsection{Modelo de regresión múltiple}

El análisis de regresión múltiple refleja el modelo empírico para la estimación de FMC y EWT a través de las respuestas espectrales de las hojas en cada longitud de onda y para cada especie. Los presentes modelos han sido calculados con un intervalo de confianza del 95\%, previo a su uso y generalización se deberían incluir más mediciones como parámetros de entrada, de manera que permitieran obtener un mejor ajuste. El valor del nivel crítico $(\mathrm{p}<0.01)$ es igual para todos los modelos, lo que indica que sí existe relación lineal significativa y, por tanto, se puede afirmar que los modelos empíricos para FMC y EWT ofrecen un buen ajuste a la nube de puntos (Figs. 8 y 9). 

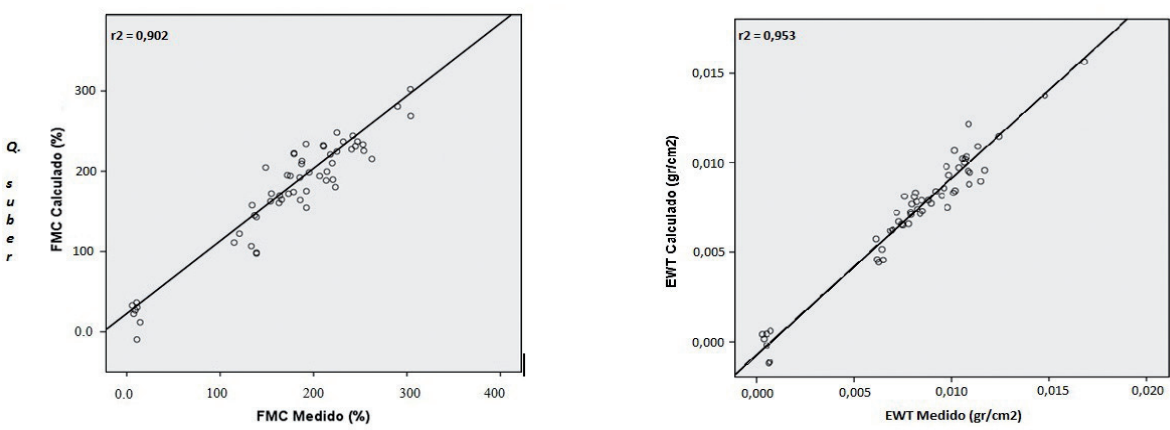

Figura 8: Relación entre valores estimados y observados para Q. suber.
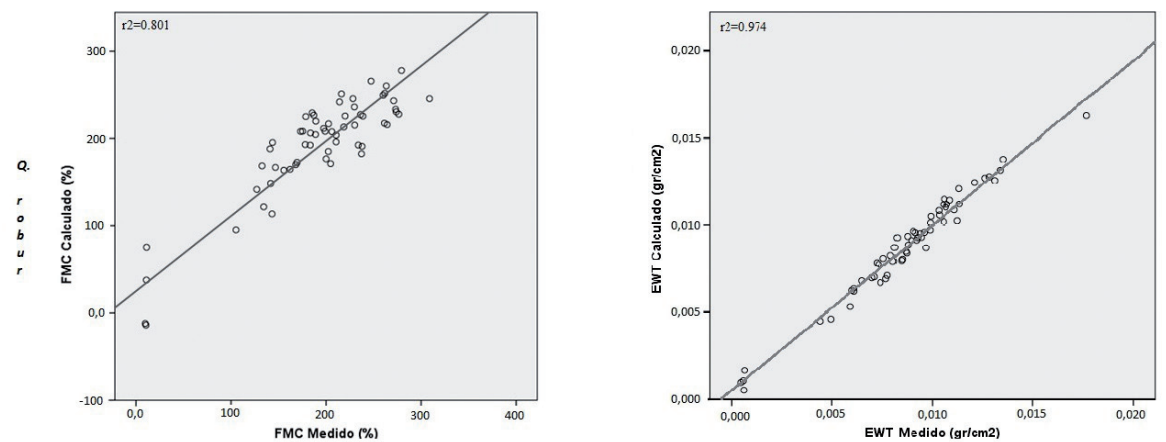

Figura 9: Relación entre valores estimados y observados para Q. robur.

El modelo para estimar el FMC del Q. suber tendría la siguiente función:

$$
\begin{gathered}
\text { FMC = 235.331 }-15128.383 \rho_{(1449.09)}-6407.677 \rho_{(1395.80)}+4885.365 \rho_{(1667.13)}+ \\
16486.284 \rho_{(1435.78)}
\end{gathered}
$$

Con un valor de $r^{2}=0.902$ (corregido $r=0.894$ ).

Para el EWT de la misma especie, el modelo sería:

$$
\begin{gathered}
\mathbf{E W T}=0.010-0.166 \rho_{(1382.47)}+0.120 \rho_{(1667.13)}+0.196 \rho_{(1169.68)}-0.737 \rho_{(1498)}+ \\
0.423 \rho_{(1520.22)}+0.371 \rho_{(1462.43)}-0.216 \rho_{(1373.6)}
\end{gathered}
$$

Con un $\mathrm{r}^{2}=0.953$ (corregido $=0.947$ )

Por su parte, para $Q$. robur el modelo para estimar el FMC sería:

$$
\begin{gathered}
\text { FMC }=285.457+1078.902 \rho_{(1435.78)}+3411.042 \rho_{(1667.13)}-11636.686 \rho_{(1382.47)}+ \\
7155.153 \rho_{(1369.17)}
\end{gathered}
$$

Con un $\mathrm{r}^{2}=0.801$ (corregido $\left.=0.786\right)$. 
Y para el EWT de la misma especie:

$$
\begin{gathered}
\text { EWT }=0.012+0.035 \rho_{1453.55)}-0.148 \rho_{(970.71)}+0.127 \rho_{(1671.58)}+0.445 \rho_{(1010.46)}+ \\
0.717 \rho_{(1165.26)}-0.293 \rho_{(1072.34)}-0.106 \rho_{(1653)}-0.427 \rho_{(1355.84)}+0.310 \rho_{(1085.6)}-0.266 \rho_{(1067.93)}- \\
0.304 \rho_{(1045.81)}-0.174 \rho_{(1143.13)}+0.219 \rho_{(1116.57)}-0.155 \rho_{(1182.96)}
\end{gathered}
$$

Con un $\mathrm{r}^{2}=0.976$ (corregido $\left.=0.968\right)$.

\section{Discusión}

Como cabía esperar, se observó que las bandas más sensibles al contenido de agua en la planta son aquellas que se ubican en el rango de los 1450-1600 nm, debido a que la respuesta de la vegetación en estas longitudes de onda está dominada por la fuerte absorción del agua (Knipling, 1970), verificándose así la relación inversa que existe entre el contenido de agua y la reflectividad de esta zona del espectro. Resultados similares se encontraron en otros trabajos (De Santis et al., 2006; Yebra et al., 2005). La correlación directa del SWIR y el contenido de agua en la planta se debe a que el agua es un buen absorbente del flujo de radiación incidente, por lo que la cantidad de energía solar reflejada en esta región es una función inversa de la cantidad de agua presente en la hoja (Knipling, 1970).

No se pudo determinar una relación directa entre la reflectividad en el IRC y el contenido de agua en las hojas para ninguna de las dos especies, denotando así que la reflectividad en esta región del espectro está más directamente relacionada con la estructura interna de la hoja. Varios autores citan que cuando la planta se somete a estrés hídrico, el deterioro de la estructura interna causa el aumento de la reflectividad en el IRC (Vogelman y Björn, 1984), ya que la reflectividad se incrementa debido a que el índice de refracción de los materiales celulares secos es mayor que el de las células hidratadas (Carter, 1991). Sin embargo, otros autores no observaron cambios significativos en esta banda (Carter, 1991) y otro grupo detecta una reducción de la reflectividad (Westman y Price, 1988). Con el presente experimento se confirma que el deterioro de la estructura celular interna de la hoja (transpiración, fotosíntesis, productividad) es lo que afecta directamente a las variaciones de reflectividad y no el contenido de agua.

Las correlaciones con la materia seca (DMC) han sido generalmente bajas, lo que indica que para estas especies, el estrés hídrico estimulado no supone un cambio en la biomasa de la hoja. Como es sabido, diversas especies mediterráneas responden a la carestía de agua con un aumento en la biomasa, para reducir la evapotranspiración (Gratani et al., 2006).

La alta correlación entre los índices espectrales deducidos a partir de las bandas MODIS y el contenido de agua resulta de gran interés para utilizar las imágenes de este sensor en la estimación operativa del riesgo de incendios, ya que ofrece una cobertura espacial $(500 \times 500 \mathrm{~m})$ y temporal (diaria) idónea para el seguimiento de las condiciones de peligro, particularmente las relacionadas con el dinamismo hídrico del combustible vivo. Varios autores han mostrado ya las posibilidades prácticas de este enfoque (Yebra 
et al., 2013), pero en este caso se muestra con dos especies de árboles bastante extendidas en nuestro país y que previamente no se habían empleado en este tipo de modelos.

\section{Referencias bibliográficas}

Bowyer, P., Danson, F.M. 2004. Sensitivity of spectral reflectance to variation in live fuel moisture content at leaf and canopy level. Remote Sensing of Environment 92, 297-308.

Boyer, J.S. 1995. Measuring the water status of plants and soils. Academic Press, Inc, Londres.

Burgan, R.E., Rothermel, R.C. 1984. BEHAVE : fire behavior prediction and fuel modeling system -- FUEL subsystem. USDA Forest Service, Intermountain Forest and Range Experiment Station, $126 \mathrm{pp}$.

Camia, A., Leblon, B., Cruz, M., Carlson, J.D., Aguado, I. 2003. Methods Used to Estimate Moisture Content of Dead Wildland Fuels. En Wildland Fire Danger Estimation and Mapping. The Role of Remote Sensing Data, E. Chuvieco (Ed.), World Scientific Publishing, Singapore, pp. 91-117.

Carter, G.A. 1991. Primary and secondary effects of water content on the spectral reflectance of leaves. American Journal of Botany 78, 916-924.

Castro, F.X., Tudela, A., Sebastiá, M.A. 2003. Modeling moisture content in shrubs to predict fire risk in Catalonia (Spain). Agricultural and Forest Meteorology 116, 49-59.

Ceccato, P., Leblon, B., Chuvieco, E., Flasse, S., Carlson, J.D. 2003. Estimation of Live Fuel Moisture Content. En Wildland Fire Danger Estimation and Mapping. The Role of Remote Sensing Data, E. Chuvieco (Ed.), World Scientific Publishing, Singapore, pp. 63-90.

Chuvieco, E., Cocero, D., Riaño, D., Martín, M.P., Martínez-Vega, J., de la Riva, J., Pérez, F. 2004. Combining NDVI and Surface Temperature for the estimation of live fuel moisture content in forest fire danger rating. Remote Sensing of Environment 92, 322-331.

De Santis, A., Vaughan, P., Chuvieco, E. 2006. Foliage moisture content estimation from 1-D and 2-D spectroradiometry for fire danger assessment. Journal of Geophysical Research Biosciences 111, doi:10.1029/2005JG000149.

Gao, B.C. 1996. NDWI. A normalized difference water index for remote sensing of vegetation liquid water from space. Remote Sensing of Environment 58, 257-266.

Gratani, L., Covone, F., Larcher, W. 2006. Leaf plasticity in response to light of three evergreen species of the Mediterranean maquis. Trees-Structure and Function 20, 549-558.

Jurdao, S., Oliva, P., Yebra, M., Chuvieco, E. (enviado). Leaf and canopy response to plant drying: implications to estimate live fuel moisture content from Radiative Transfer Models. Photogrammetric Engineering and Remote Sensing.

Knipling, E.B. 1970. Physical and Physiological basis for the reflectance of visible and nearinfrared radiation from vegetation. Remote Sensing of Environment 1, 155-159.

Mendez, R., Molinero, F. (eds.) 1993. Geografía de España. Ariel, Barcelona.

Ruiz de la Torre, J. (ed.) 1990-1999. Mapa Forestal de España. Ministerio de Medio Ambiente, Madrid.

Vogelman, T.C., Björn, L.O. 1984. Measurement of light gradients and espectral regime in plant tissue with a fiber optic probe. Physiologia Plantarum 60, 361-368.

Westman, W.E., Price, C.V. 1988. Spectral changes in conifers subjected to air pollution and water stress: experimental studies. IEEE Transactions on Geoscience and Remote Sensing 26, 11-20.

Yebra, M., Chuvieco, E. 2009. Linking ecological information and radiative transfer models to estimate fuel moisture content in the Mediterranean region of Spain: Solving the ill-posed inverse problem. Remote Sensing of Environment 113, 2403-2411. 
Yebra, M., de Santis, A., Chuvieco, E. 2005. Estimación del peligro de incendios a partir de teledetección y variables meteorológicas: variación temporal del contenido de humedad del combustible". Recursos Rurais 1, 9-19.

Yebra, M., Chuvieco, E., Riaño, D. 2008. Estimation of live Fuel Moisture Content from MODIS images for fire risk assessment. Agricultural and Forest Meteorology 148, 523-536.

Yebra, M., Dennison, P., Chuvieco, E., Riaño, D., Zylstra, P., Hunt, E.R., Danson, F.M., Qi, Y., Jurdao, S. 2013. A global review of remote sensing of live fuel moisture content for fire danger assessment: moving towards operational products. Remote Sensing of Environment $136,455-468$. 